\title{
Questionnaire to measure perceived symptoms and disability in asthma
}

\author{
T P Usherwood, A Scrimgeour, J H Barber
}

\begin{abstract}
A questionnaire was designed for completion by parents of asthmatic children aged from 5-14 years that provides scales of good content validity and internal reliability for the measurement of perceived disability, perceived nocturnal symptoms, and perceived daytime symptoms. We hope that this questionnaire will be included in future clinical studies of these patients.
\end{abstract}

Questionnaires addressed to parents are widely used to measure symptoms and disability in children with asthma, ${ }^{12}$ but there is no accepted, standard one in use.

A number of formal procedures have been described for developing a questionnaire that will provide one or more quantitative measures of the effects that may be experienced by a person with a chronic disease. ${ }^{3}$ These procedures have much in common both with each other and with the methods described for the development of multidimensional health indexes such as the Nottingham Health Profile. ${ }^{4} \mathrm{~A}$ wide range of statements is generated, each of which describes an effect of the disease that the patient might experience. In the case of a disease that affects children, statements may be generated about how parents perceive the effects of the disease. These statements are refined by discussion with patients, or with their parents, and are then incorporated into a questionnaire.

A survey is undertaken using the questionnaire, and the responses to each item are scored. Those groups of items that exhibit highly correlated responses are identified and inspected. If they all refer to similar aspects of the patient's experience then they may be considered to represent a scale that measures a single dimension of that experience. An individual respondent's scores on the items of the scale may then be added together to produce a single summary score that indicates his or her position on that dimension. Usually it is enough to add the scores without weighting them. ${ }^{6}$ Obviously, the questionnaire may contain more than one group of highly correlated items, in which case-if each group refers to a different aspect of experience of the disease-then each group will provide a scale that measures a different dimension of experience.
Our aim was to develop a questionnaire to be completed by parents of asthmatic children aged 5 to 14 years inclusive to provide quantitative measures of the symptoms and disabilities that they perceived in their children.

\section{Methods}

DEVELOPMENT OF THE QUESTIONNAIRE

Four experienced general practitioners generated 34 descriptions of how asthma might affect a child. Several of these descriptions were duplicates, and were therefore combined, yielding a list of 26 statements. Each statement was presented on the first draft of the questionnaire with five possible responses ranging from 'Every day' to 'Not at all' (table 1). The four statements that referred to nocturnal symptoms were given responses ranging from 'Every night' to 'Not at all'. So that statements concerning problems at school would make sense even if the questionnaire was administered towards the end of the summer holidays, it was introduced by the statement that it referred to the preceding three months.

This draft questionnaire was discussed with several mothers of asthmatic children. The first mother to be interviewed was asked to complete the questionnaire, discussing each item in turn with one of the authors (TPU). She was asked to explain her interpretation of each descriptive statement, and why she chose a particular response. She was also asked if she found the wording of any phrase confusing or unclear. After this a new draft questionnaire was produced and a similar interview was carried out with a second mother. This process was continued until a total of 16 mothers had been interviewed, although no changes were considered necessary in the light of comments made by the last seven. By this time the number of questions had been reduced to the 17 statements listed in table 2, each with its five possible responses.

\section{SURVEYS}

Two surveys were then undertaken with the 17 item questionnaire. For survey A, children were recruited who had been entered by their general practitioners into a multicentre trial of an inhaled steroid for the treatment of asthma.

Table 1 Example of item from initial draft questionnaire (also used in the final version) 
Table 2 The 17 descriptive statements from the questionnaire. " The statements were preceded by the phrase 'Over the past three months'

\begin{tabular}{ll}
\hline Item No & Descriptive statement \\
\hline 1 & Your child has been wheezy during the day \\
2 & Your child has coughed during the day \\
3 & Your child has complained of being short of breath \\
4 & Your child has complained of a pain in the chest \\
5 & Exertion (eg, running) has made your child breathless \\
6 & Your child has stayed indoors because of wheezing or coughing \\
7 & His/her asthma has stopped your child from playing with his or her friends \\
8 & During term time, your child's education has suffered due to his or her asthma \\
9 & Asthma has stopped your child from doing all the things that a boy or girl should at his or her age \\
10 & Your child's asthma has interfered with his or her life \\
11 & Asthma has limited your child's activities \\
12 & Taking his or her inhaler or other treatment has interrupted your child's life \\
13 & Your child's asthma has limited your activities \\
14 & You have had to make adjustments to family life because of your child's asthma \\
15 & Your child has coughed at night \\
16 & Your child's sleep has been disturbed by wheezing or coughing \\
17 & Your child has been woken up by wheezing or coughing \\
\hline
\end{tabular}

${ }^{*}$ Copyright T P Usherwood, 1988; see editors' note on p781.

They had all had asthma throughout the year, and either had symptoms despite their current medication, or were using an inhaled bronchodilator at least three times a day. Entry was delayed if the child had an acute respiratory tract infection. Parents completed the questionnaire at the time of their child's entry into the trial.

For survey B, general practitioners in five practices were asked to distribute the questionnaire to the parents of any asthmatic child aged from 5 to 14 years with whom they came into contact over a three week period. The covering letter asked the parents to complete the questionnaire and to return it in an attached selfaddressed envelope. The questionnaires were returned anonymously, and no further details about the child were requested.

\section{ANALYSIS}

The data from survey $A$ were analysed by the method described by McKennell. ${ }^{6}$ Before analysis the responses to each item of the questionnaire were coded from zero for 'Not at all' in unit steps up to four for 'Every day' or 'Every night'. Product moment correlation coefficients were then calculated between the responses to each pair of items in the questionnaire. Principal component analysis with varimax rotation was carried out on the correlation matrix to identify a small number of groups of items, each of which seemed to deal with a single aspect of the effects of the disease. The internal reliability of each resulting scale was assessed by calculating coefficient $\alpha$, a measure of mean interitem correlation. A value of 0.7 or more was deemed acceptable. ${ }^{7}$ As a check to ensure that the scales derived from survey A did not have spuriously high mean interitem correlations (perhaps as a result of the recruitment criteria for that survey) coefficient $\alpha$ was also calculated for each scale from the data from survey $B$.

\section{Results}

One hundred and sixty four copies of the 17 item questionnaire were returned by parents of children in survey $A$. The mean (SD) age of the children was $9 \cdot 8(2 \cdot 7)$ years, and 108 of the children (66\%) were boys. Five principal compo- nents were extracted in the initial analysis. Inspection of the group of statements that correspond to each component, however, suggested a degree of overlap in the content of the dimensions measured. For example 'Exertion has made your child breathless' was found in one group, but 'Your child has complained of being short of breath' occurred in a different group.

It was noted that statement 12 'taking his or her inhaler or other treatment has interrupted your child's life' was answered 'not at all' by 114 of the respondents $(70 \%)$. In contrast, fewer than half the respondents gave this response to any other single statement. Because of the apparent insensitivity of item 12 , therefore, it was excuded, and principal component analysis repeated. The results of this analysis are shown in table 3, from which it can be seen that four components were extracted. On inspection, the first component seemed to measure disability, the second measured nocturnal symptoms, the third daytime symptoms, and the fourth consisted mainly of a single item enquiring about chest pain. The first three components extracted were considered to identify usable scales of good content validity, which also exhibited acceptable values of coefficient $\alpha$ (table 4). The fourth identified only one item, so that no scale could be constructed from this.

Table 3 Results of principal component analysis with varimax rotation on the data from survey $A$, after exclusion of item 12

\begin{tabular}{lll}
\hline $\begin{array}{l}\text { Principal } \\
\text { component }\end{array}$ & $\begin{array}{l}\text { Main } \\
\text { contributing } \\
\text { items }\end{array}$ & $\begin{array}{l}\text { Variance } \\
\text { explained } \\
\text { (cumulative \%) }\end{array}$ \\
\hline First & $6,7,8,9,10,11,13,14$ & 38 \\
Second & $15,16,17$ & 50 \\
Third & $1,2,3,5$ & 59 \\
Fourth & 4 & 66 \\
\hline
\end{tabular}

Table 4 Values of coefficient $\alpha$ calculated for each scale from surveys $A$ and $B$

\begin{tabular}{lll}
\hline Scale & \multicolumn{2}{l}{ Coefficient $\alpha$} \\
\cline { 2 - 3 } & Survey $A$ & Survey $B$ \\
\hline Disability & 0.87 & 0.88 \\
Nocturnal symptoms & 0.88 & 0.92 \\
Daytime symptoms & 0.71 & 0.76 \\
\hline
\end{tabular}


Table 5 Scores obtained from each scale from surveys $A$ and $B$

\begin{tabular}{lll}
\hline Scale & \multicolumn{2}{l}{ Median (range) score } \\
\cline { 2 - 3 } & Survey $A$ & Survey $B$ \\
\hline Disability & $9(0-23)$ & $3(0-20)$ \\
Nocturnal symptoms & $6(0-12)$ & $5 \cdot 5(0-12)$ \\
Daytime symptoms & $9(1-15)$ & $6(0-14)$ \\
\hline
\end{tabular}

Between 60 and 70 copies of the 17 item questionnaire were distributed to the parents of asthmatic children in survey $B$, and 55 copies were returned. Fifty two of these had been completed in full. Coefficient $\alpha$ was again calculated from this set of data for each of the first three scales derived above. The results are given in table 4; the values obtained were similar to those obtained previously.

To calculate single scores for an individual child for each of the three scales, the unweighted scores for each item in each scale were simply added together, as explained earlier. ${ }^{5}$ Thus the disability score for any one patient lay between 0 and 32 inclusive, the nocturnal symptom score lay between 0 and 12 inclusive, and the daytime symptom score lay between 0 and 16. In each case the higher the score the greater the perceived disability or extent of symptoms. Table 5 gives the median and range of scores for each scale from each survey. Taking the results of both surveys, the observed scores were distributed throughout most of the potential range of the disability scale, the whole of the nocturnal symptom scale, and most of the daytime symptom scale.

We concluded that the three scales defined by the first three principal components in table 3 were each of sufficient content validity and internal reliability to provide useful measuring instruments for perceived disability, perceived nocturnal symptoms, and perceived daytime symptoms, respectively.

\section{Discussion}

The questionnaire described in this report is simple and takes parents only a few minutes to complete. Scores that represent the child's position on three important dimensions of perceived morbidity may be calculated rapidly. Clearly parents' perceptions are only a proxy for their children's experiences. Much paediatric research, however, as well as clinical practice, has to depend on parents' reports. An exception is provided by the availability of overnight tape recording to determine the severity of nocturnal coughing. Archer and Simpson reported that the frequency of nocturnal coughing did not correlate with the nocturnal cough scores that were recorded by parents in a symptom diary using an ordinal scale from 0 (no cough) to 3 (severe cough). ${ }^{8}$ Thus results obtained from parents were a poor proxy for this aspect of their children's experience. Validation of this questionnaire by direct observation over a three month period would not be practicable, neither is it safe to assume that parents' records in a symptom diary would provide a gold standard with which to compare the questionnaire. As with any proxy instrument, however, evidence supporting or rejecting the construct validity of its derived scales will accrue with further experience of its use. So far the three scales seem to have good content validity and good internal reliability.

Of course the three scales have only ordinal (ranking) measurement properties, so their observed frequency distributions in the two survey samples have been summarised in table 5 using non-parametric descriptive statistics. Although they were adequate for the present aim, neither of the samples was selected at random from an identified population so the summary statistics in table 5 should not be taken as representative of underlying population characteristics.

Although designed primarily as a research instrument, the questionnaire may be useful in the consulting room. The scales provide three measures of parents' perceptions of the recent state of a child's asthma that may be used to complement respiratory function tests and the clinician's usual questions. Furthermore, the answers to individual items of the questionnaire may alert the clinician to otherwise unexpected problems. A high score in question 8 (education), for example, might prompt further discussion about difficulties at school. To simplify the calculation of scores during the consultation, items 4 and 12 may be omitted from the questionnaire. The first four items then constitute the daytime symptom scale, the next eight items the disability scale, and the final three items the nocturnal symptom scale.

We believe on the evidence obtained so far that this questionnaire is a useful addition to existing measures of the effects of asthma on children of school age. We hope that it will be included among the outcome measures in future clinical studies of the condition. A copy of the questionnaire can be obtained from TPU (stamped

Editors' envelope please). Editors' note: the questionnaire is not the copyright of the
Archives of Disease in Childhood but of TPU; permission for reproduction should be obtained from Dr T P Usherwood Department of General Practice, Medical School, Beech Hil Road, Sheffield S10 2RX.

1 Anderson HR, Bailey PA, Cooper JS, Palmer JC, West S. Morbidity and school absence caused by asthma and whee ing illness. Arch Dis Child 1983;58:777-84.

2 Hill RA, Standen PJ, Tattersfield AE. Asthma, wheezing and school absence in primary schools. Arch Dis Child $1989 ; 64: 246-51$.

3 Guyatt GH, Bombardier C, Tugwell PX. Measuring disease specific quality of life in clinical trials. Can Med Assoc $\mathcal{J}$ 1986;134:889-95.

4 Hunt SM, McEwen J, McKenna SP. Measuring health status. London: Croom Helm, 1986.

5 Armitage P, Berry G. Statistical methods in medical research. 2nd Ed. Oxford: Blackwell, 1987.

6 McKennell A. Attitude measurement: use of co-efficient alpha with cluster or factor analysis. Sociology 1970;4 $227-45$

7 Abramson JH. Survey methods in community medicine. 3rd Ed. Edinburgh: Churchill Livingstone, 1984.

8 Archer LNJ, Simpson H. Night cough counts and diary card scores in asthma. Arch Dis Child 1985;60:473-4. 